\title{
Invasive FFR - Current Applications and New Developments
}

\author{
Monica Chițu1, Nora Rat¹, Diana Opincariu², Alexandra Stănescu², András Mester², Theodora \\ Benedek ${ }^{1,2}$, Imre Benedek ${ }^{1,2}$ \\ ${ }^{1}$ Center of Advanced Research in Multimodality Cardiac Imaging, Cardio Med Medical Center, Tîrgu Mureș, Romania \\ 2 University of Medicine and Pharmacy, Tîrgu Mureș, Romania
}

\section{CORRESPONDENCE}

\section{Nora Rat}

Str. Gheorghe Marinescu nr. 38

540139 Tîrgu Mureș, Romania

Tel: +40 265215551

E-mail: ratnora@gmail.com

\section{ARTICLE HISTORY}

Received: 5 October, 2016

Accepted: 3 November, 2016
Monica Chițu • Str. Gheorghe Marinescu nr. 38, 540139 Tîrgu Mureș, Romania. Tel: +40 265215551. E-mail: iuliachitu@yahoo.com

Diana Opincariu • Str. 22 Decembrie 1989 nr. 76, 540124 Tîrgu Mureș, Romania. Tel: +40 265217333. E-mail: diana.opincariu@yahoo.ro

Alexandra Stănescu • Str. 22 Decembrie 1989 nr. 76 540124 Tîrgu Mures, Romania. Tel: +40 265217333 E-mail: alexandrastanescu90@gmail.com

András Mester • Str. 22 Decembrie 1989 nr. 76 540124 Tîrgu Mureș, Romania. Tel: +40 265217333. E-mail: andras.mester@yahoo.com

Theodora Benedek • Str. Gheorghe Marinescu nr. 38 540139 Tîrgu Mureș, Romania. Tel: +40 265215551. E-mail: theodora.benedek@gmail.com

Imre Benedek • Str. Gheorghe Marinescu nr. 38, 540139 Tîrgu Mureș, Romania. Tel: +40 265215551 E-mail: imrebenedek@yahoo.com

\begin{abstract}
Myocardial ischemia caused by coronary atherosclerosis is the main cause of cardiovascular mortality, which is the first cause of death in developed countries. Inducible myocardial ischemia is a negative prognostic factor for coronary artery disease patients, and it represents a major risk stratification marker for predicting mortality. The evolution of these patients depends largely on the extension of the ischemic myocardial mass and the severity of the inducible ischemia. Most patients do not show angiographically significant coronary stenosis. Therefore, such patients do not undergo coronary revascularization therapies, even though they present functional stenoses that trigger myocardial ischemia under stress conditions, which subsequently lead to a high risk for major adverse cardiovascular events. We performed a review that aims to pinpoint invasive techniques used for evaluating the functional impact of a coronary lesion that is considered non-significant upon angiographic examination, but which triggers episodes of myocardial ischemia under stress conditions, and to describe the functional markers that show the highest specificity for predicting ischemic risk, in order to recommend invasive coronary revascularization.
\end{abstract}

Keywords: invasive fractional flow reserve, inducible myocardial ischemia, functional significance of coronary stenosis

\section{BACKGROUND}

The methods currently used for myocardial revascularization, such as percutaneous coronary angioplasty and coronary artery bypass grafting, aim to reestablish the optimal circulatory supply to the myocardial tissue, thus reducing myocardial ischemia. Inducible myocardial ischemia is a negative prognostic factor and a predictor for the rate of major adverse cardiac events (MACE) in patients with coronary atheromatous lesions. ${ }^{1}$ Several studies have shown that coronary revascularization in patients with inducible ischemia is superior in efficiency compared to pharmacological treatment. ${ }^{2,3}$ Moreover, patients with coronary lesions that do not trigger ischemia have a better outcome and evolution under pharmacological treatment, with a complication rate of less than $1 \%$. Identifying coronary lesions that cause episodes of myocardial ischemia is 
essential in the management of coronary patients, and the use of various techniques for assessing the extension and localization of the ischemic myocardium is of essence in therapeutic decision-making and prognostic evaluation.

Currently, detection of myocardial ischemia is performed using different stress-test methods (ECG stress test, stress echocardiography, myocardial stress imaging). Despite their widespread use, these methods hold several limits, including the relatively low specificity and sensitivity in the identification of the ischemic myocardial territory, especially in patients with multi-vessel coronary atherosclerosis. Nuclear imaging methods are considered to have a higher specificity regarding the location of stressinduced ischemia, however, in case of multiple coronary stenotic lesions, they present a limited sensitivity due to the absence of a non-ischemic myocardial region with normal perfusion that would act as reference. ${ }^{4,5}$

\section{PHYSIOLOGY OF CORONARY CIRCULATION}

Coronary flow is dependent on the epicardial artery system, as well as the collateral vascular network and the microcirculation, which work together in order to optimize the myocardial oxygen supply.

Hemodynamic evaluation of the trans-lesional gradient in stress conditions led to significant improvements in the clinical approach and reevaluation of the indication for revascularization in patients with non-significant coronary lesions upon invasive coronary angiography examination (the gold-standard method for evaluating the degree of stenosis), but which exhibit stress-induced myocardial ischemia. The angiographic evaluation of coronary lesions that are considered borderline, but which have been demonstrated to be associated with inducible stress ischemia, has several limits resulting from intra- and interprocedural variability, as well as a limited spatial resolution in certain coronary territories. ${ }^{6,7}$

Myocardial ischemia is triggered by an unbalance between the myocardium oxygen request and supply. Oxygen demand is influenced by wall stress, myocardial contractility and heart rate, while the supply is provided by the arterial oxygen concentration and the coronary flow. Coronary flow is calculated with the Poisseuille equation, as the linear relationship between coronary perfusion pressure and vascular resistance. The latter is dependent exclusively on the microvascular system in lack of any coronary lesions, and on the degree of myocardial compression (situations such as cardiac hypertrophy). ${ }^{8}$ Coronary flow is autoregulated by arterial vasodilatation when the coronary pressure decreases, being dependent on the intraaortic pres- sure. When the autoregulation mechanism is exceeded, the affected myocardial territory becomes ischemic. The adenosine test is based on inducing coronary arteriolar vasodilatation and decreasing microvascular resistance, reaching the point where the autoregulation mechanism is exceeded. Thus, the local hyperemia will unmask the ischemic myocardial territory by increasing the transstenotic intracoronary gradient. ${ }^{9,10}$

\section{METHODS TO EVALUATE THE FUNCTIONAL SIGNIFICANCE OF CORONARY LESIONS}

\section{Determination of Fractional Flow Reserve (FFR)}

Coronary fractional flow reserve (FFR) is determined through maximum coronary vasodilatation in a narrowed coronary sector (due to atherosclerotic lesions), in order to achieve an optimal mechanical work for a certain level of effort. FFR is granted by an increased flow in the collateral coronary system and in the arteriolar microcirculation. The index of FFR, the numerical value of FFR, is determined as the ratio between the maximal blood flow in a coronary sector and the maximal coronary pressure in optimal conditions, based on the intra-aortic pressure, in the same conditions of induced vasodilation. ${ }^{11}$

The invasive determination of FFR represents the gold standard procedure for assessing the functional significance of coronary stenoses that are considered as "triggers" for ulterior coronary revascularization. FFR can offer the interventional cardiologists essential information in the decision-making process regarding myocardial revascularization therapies. This method has the advantage of offering an optimal vascular spatial resolution and a high reproducibility, since it is capable of reflecting the global myocardial flow (from the epicardial vessels, as well as from the microcirculation), and it does not depend on hemodynamic variations. The determination of FFR together with newer methods of assessing the morphology of atheromatous plaques, such as optical coherence tomography (OCT) and virtual histology ultrasound ( $\mathrm{VH}-$ US), creates a complex model of imaging analysis of the coronary plaque, including a descriptive analysis of the coronary plaque (fibrous cap, lipidic necrotic core, calcification degree) together with assessment of its functional significance (by calculating the translesional gradient as an expression of a decreased flow distally from the lesion).

Randomized trials that have used the assessment of the functional significance of coronary stenoses have shown that using FFR in the revascularization algorithm has led to 
a reduction in mortality and MACE rates in patients with CAD. The Fractional Flow Reserve versus Angiography for Multivessel Evaluation (FAME) study, which evaluated the routine use of FFR in comparison with traditional coronary angiography in establishing the indication for elective coronary angioplasty in multivessel CAD, showed that the angiographic estimation of coronary lesion severity is inadequate in the assessment of functional significance, both in stenoses of $50-70 \%$, as well as those of $70-90 \% .^{12,13}$

The invasive measurement of the coronary flow reserve is based on crossing the stenosis with a floppy guidewire that is connected to a pressure sensor placed $3 \mathrm{~cm}$ from the tip; the pressure curve is recorded through the catheter and is shown in a digital format. The pressure curve recordings are compared with the intraaortic pressure. Because, technically, this procedure is difficult to perform for detecting the actual coronary flow, the method has been adapted by administering a vasodilator agent, which is adjusted to the caliber of the coronary artery in question, triggering a maximal coronary dilation, both on the epicardial and microvessels (the vascular resistance system). The vasodilator agents that have been established for the use of FFR determination include: adenosine (dose of 140 $\mu \mathrm{g} / \mathrm{kg} / \mathrm{min}$ via a peripheral venous line, $40 \mu \mathrm{g}$ in the right coronary artery, $40-80 \mu \mathrm{g}$ in the left coronary artery), isosorbide dinitrate $(200 \mu \mathrm{g}$ intracoronary), papaverine (10 $\mu \mathrm{g}$ in the right coronary artery, $15-20 \mu \mathrm{g}$ in the left coronary artery). By annulling the arterial coronary resistance both in the epicardial and microvascular systems, a linear relationship between the coronary flow and the coronary pressure will occur. Therefore, the coronary FFR assessment is determined, in clinical practice, by calculating the ratio between the medium pressure measured distally from the coronary stenosis, and the intraaortic pressure. ${ }^{14,15,16}$

$$
\text { FFR myo }=\text { Pd } / \text { PAo }
$$

The variable resulted from comparing the translesional pressure gradient variations in conditions of maximum hyperemia, reflects the maximum coronary flow that can be obtained in similar conditions as those occurring under stress situations; furthermore, the obtained variable is a measurement of the myocardial capacity to perform its function without paying the price of myocardial ischemia. The determination of this ratio is easily performed in the catheterization laboratory, and can be applied in daily practice. The standard value at which the coronary flow is considered optimal is characterized by a coronary FFR of 1.0 , which means that in the absence of any coronary stenoses, the intracoronary pressure is unmodified all the way to the peripheral region of the vessel. An FFR value of $<1.0$ is illustrating that there is an incomplete capacity to fully increase the coronary flow when the cardiac workload requires it. There are several circumstances when the maximal vasodilation is altered, decreasing the sensitivity of the method. Such circumstances could be represented by STEMI or non-STEMI myocardial infarctions, conditions associated with a marked endothelial dysfunction at the level of the microcirculation, or cardiac hypertrophy that causes extrinsic compression of the coronary arteries. ${ }^{8,17,18}$

All in all, the method has been validated in clinical trials as being useful in evaluating all the borderline coronary stenoses. Studies have proven that a cut-off value for FFR of $0.75-0.8$ is highly specific for inducible myocardial ischemia, and it is directly correlated with the extension of the myocardial ischemia as assessed through nuclear imaging methods, as well as with the functional angina class and prognosis of the coronary patient. An FFR value of 0.75 shows that the coronary flow can be increased with maximum $75 \%$ over the normal value in conditions of physical stress or effort.

The limited variability of the cut-off value $(0.75-0.8)$, which can categorize a coronary stenosis as being hemodynamically significant, leads to several comments regarding this interval, which is considered as a "grey zone" in the decision-making process of coronary revascularization. The experts' opinion is that the decision-making should consider the size of the ischemic myocardial area, as shown by noninvasive tests (stress echocardiography, nuclear imaging techniques). In other words, the indication for percutaneous coronary intervention (PCI) in such cases, will be established taking into consideration the anatomical distribution of the ischemic territory, the severity of the ischemia occurred during stress tests, the patient's adherence to antiplatelet therapy, especially if the patients have a high risk for ischemia, such as diabetics, subjects with left-main lesions or diffuse coronary atherosclerotic involvement, as well as individuals with previous thrombotic events. ${ }^{19-22}$

Studies have shown that there are several special circumstances in which the use of FFR requires caution in interpretation, either due to lesions that have a vital risk, or due to interpretation errors that may occur. Such circumstances are listed below:

a) Evaluation of the functional significance of anterograde and retrograde collateral vessels, to determine which lesion should be re-vascularized (stent or balloon angioplasty) for reducing the ischemic myocardial mass. ${ }^{23}$

b) Left main coronary artery lesions, which are rarely single lesions; most frequently, left main lesions are 
associated with severe stenoses involving the left anterior descendant (LAD) or circumflex artery (CxA), which causes an increased FFR value at the level of the left main artery (higher values if the associated lesions are more proximal). Despite this, the European guideline for elective myocardial revascularization states that the left main atherosclerotic lesions should be revascularized if the myocardial ischemia is inducible via noninvasive stress testing, if the FFR is $<0.8$, or if the stenosis is angiographically estimated at $50-90 \% .^{24}$

c) Performing stent angioplasty for bifurcation lesions with provisional stenting over the secondary branch leads to plaque protrusion in the secondary branch ostium, as assessed through coronary angiography. Even if in most cases ostium stenotic lesions of lateral branches appear as significant upon coronarographic examination, methods of simultaneous dilation of the main vessel and lateral vessel are not justified. Performing FFR determination in such lesions should be followed by a "kissing" angioplasty only if the FFR is $<0.75$; otherwise the associated risks (such as stent fracture) are not acceptable. Koo et al. showed that using FFR assessment in the interventional approach of bifurcation lesions significantly reduces the rate of complications that may occur in such complex lesions. ${ }^{25,26}$

d) Sequential coronary atherosclerotic involvement and diffuse coronary atherosclerosis is a challenge for the interventional cardiologist; identifying the lesion with the maximum impact on myocardial ischemia with FFR by using a continuous pull-back maneuver with simultaneous administration of a vasodilator agent leads to reduction of the absolute of risk for major adverse cardiovascular events. The Fractional Flow Reserve versus Angiography for Multivessel Evaluation (FAME) trial that included 1,005 patients with multiple vascular CAD has shown that FFR-guided PCI can reduce the absolute ischemic risk by $5 \% .27$

e) In ST-elevation myocardial infarction (STEMI), evaluation of the functional significance of the culprit lesion is not relevant; the patient undergoes coronary angioplasty in spite of the lack of hemodynamic significance of the coronary lesion; FFR measurement in such cases is altered due to the thrombotic content of the lesion (distal embolization, myocardial stunning, acute ischemic micro-vascular dysfunction that occurs in the no-reflow phenomenon). It is recommended that FFR measurements should be perfor- med after approximately 5 days following the acute event, when the vascular abnormalities become stable. Nalianis et al. have shown that early revascularization of non-culprit lesions in acute STEMI, guided by FFR measurements, reduces short and long term ischemic complications and death rates. ${ }^{28}$

Clinical trials, including FAME and DEFER, have shown that revascularization of a coronary stenosis with a measured FFR of $<0.75 \%$ has led to a decrease in the 1-year and 5-year rates of MACE, and that subjects with an FFR of $>0.75 \%$ presented a low percentage of composite endpoints at 1 and 5 years (including myocardial infarction, angina symptoms and the use of antianginal therapies). At the same time, the cost-efficiency ratio evaluated by the rate of re-hospitalizations is optimal, thus the 2014 guidelines for revascularization include as a class I indication the use of FFR measurements in the establishing of the functional significance of coronary stenoses, before commencing to stress testing, and class IIa indication of FFR measurements for patients with multi-vessel coronary artery disease in establishing the indication for revascularization of lesions that are angiographically estimated at $50-70 \% .{ }^{17,29,30}$

There are several undergoing trials that use FFR measurements, such as FAME 3, which compares PCI and coronary artery bypass grafting (CABG) in multi-vessel CAD patients, and which follows the rate of reinfarction, death, stroke and repeated revascularization at 1 year. SYNTAX II trial compares treatment options for multi-vessel CAD with the use of FFR; the MITNECB5 trial compares the sensitivity of FFR with various imaging methods (stress echocardiography, perfusion magnetic resonance imaging, SPECT with technetium $99 \mathrm{~m}$, or perfusion CT angiography). The FFR or OCT Guidance to RevasculariZe Intermediate Coronary Stenosis Using Angioplasty (FORZA) trial, which includes patients with intermediate coronary stenoses and compares FFR-guided PCI versus OCT-guided PCI in revascularization of complex coronary lesions estimated as intermediate upon coronary angiography. ${ }^{31}$

\section{Determination of coronary flow velocity reserve}

The coronary flow velocity reserve is a Doppler method used on a low scale before the development of FFR, which measured the coronary flow velocity, and was used for the assessment of microvascular dysfunction. This method has been proved to be influenced by hemodynamic variations and has not been validated for use in clinical practice. ${ }^{32}$ 


\section{Determination of Instantaneous Wave-Free ratio (iFR)}

iFR is a similar method as FFR, dedicated to evaluating the role of microcirculation in the genesis of myocardial ischemia and does not use vasodilator agents. The method has been created following the presumption that the coronary vascular resistance is lowest during the end-diastolic period, and it may be considered as equivalent to the value obtained during maximum myocardial hyperemia. The ADVISE study (Adenosine Vasodilator Independent Stenosis Evaluation) has shown that there is a statistically significant correlation between FFR and iFR $(\mathrm{R}=0.91$, $\mathrm{p}$ $<0.0001$ ), and that an iFR cut-off value of 0.9 is equivalent to an FFR value of $0.80 .{ }^{33}$ Comparative studies between FFR and iFR, performed with the pull-back technique in coronary arteries with diffuse atherosclerotic involvement, is highly specific, and it allows reductions in the number of implanted stents, as well as a decrease in length for stents, subsequently decreasing the risk for thrombotic events. ${ }^{34}$ The ADVISE II trial recommends that at an iFR value of $<0.86$, PCI should be performed, and at an iFR value of $>0.94$, pharmacological therapy should be initiated. ${ }^{35,36}$

The undergoing DEFINE REAL trial, which has enrolled over 3,000 patients, aims to follow-up the predictive capacity of iFR in determining the effort-induced ischemia in comparison with FFR, which is still considered gold-standard for the evaluation of functional significance of coronary atherosclerotic lesions. ${ }^{37}$ Moreover, the DEFINE FLAIR trial (NCT02053038) will randomize 2,500 patients to evaluate FFR-guided PCI versus iFR-guided PCI. Also, the PROSPECT II (A Multicenter Prospective Natural History Study Using Multimodality Imaging in $\mathrm{Pa}-$ tients With Acute Coronary Syndromes - NCT02171065) and ORBITA (Defining a Gold Standard for Ischemia: Effects of Interventional Revascularization Versus Optimum Medical Therapy on Exercise Capacity in Patients With Stable Coronary Artery Disease - NCT02062593) trials, which are still undergoing, will compare FFR and iFR in regards to efficiency for imaging atherosclerosis and related ischemia. 38,39

\section{Determination of the Index of Microvasculature Resistance (IMR)}

IMR was introduced by Fearon and is based on the determination of intracoronary pressure and temperature in hyperemia conditions, using a temperature sensor. This index is used in evaluating microvascular dysfunction, which causes angina symptoms and the occurrence of MACE in patients with no significant epicardial coronary artery stenoses. The maximal hyperemia is obtained by adenosine administrations, followed by measurements of intracoronary pressures distally form the lesion, as well as the time variation of temperatures by using the thermodilution method. Fearon et al. have shown that an IMR of $>40$ in patients following primary PCI is illustrative for the noreflow phenomenon and it is correlated with the death and re-hospitalization rates for heart failure (HR 2:1). A similar effect was shown for elective percutaneous coronary angioplasty in case of stable angina, thus suggesting the need for adequate strategies in the therapy of microvascular dysfunction both in thrombotic lesions in acute coronary syndromes, as well as in stable CAD. ${ }^{40-42}$

\section{CONCLUSIONS}

Identifying myocardial ischemia occurring in stress conditions is essential for the quantification of the ischemic risk and the prognosis of patients with coronary artery disease. A part of these patients present with multi-vessel coronary atherosclerosis and associate a series of comorbidities, which are correlated with endothelial dysfunction and micro-vessel coronary artery disease. Assessment of the functional significance of a coronary stenosis that was deemed to not have indication for revascularization upon coronary angiography examination, allows the identification of lesions that induce myocardial ischemia during maximal hyperemia (stress conditions, pharmacological agents). The combined use of FFR, IMR and iFR allows a precise detection of the ischemic potential of a coronary lesion, therefore recommending the treatment for myocardial revascularization for lesions that were initially believed to not cause significant stenosis during invasive angiographical examination.

\section{CONFLICT OF INTEREST}

Nothing to declare.

\section{ACKNOWLEDGEMENT}

This research was supported via the research grant no. 103545/2016, contract number 43/05.09.2016, entitled "High performance multimodal MRI/CT imaging platform, for applications in computational medicine, nanoparticles and hybrid imaging for the research of atherothrombotic disorders - CARDIO IMAGE", financed by the Romanian Ministry of European Funds, the Romanian Government and the European Union. 


\section{REFERENCES}

1. Shaw LJ, Berman DS, Marin DJ, et al. Optimal medical therapy with or without percutaneous coronaru interventions to reduce ischaemic burden: results from the Clinical Outcomes Utilizing Revascularization and aggressive drug Evaluation (COURAGE) trial nuclear substudy. Circulation. 2008;117:1283-1291.

2. Erne P, Schoenenberger AW, Burckhardt D, et al. Effects of percutaneous coronary interventions in silent ischaemia after myocardial infarction: The SWISS II randomized controlled trial. JAMA. 2007;297:1985-1991.

3. Pijls NH, van Schaardenburgh P, Manoharan G, et al. Percutaneous coronary intervention of functionally nonsignificant stenosis: 5-year follow up of the DEFER Study. J Am Coll Cardiol. 2007;49:2105-2111.

4. Lin GA, Dudley RA, Lucas FL, Malenka DJ, Vittinghoff E, Redberg RF. Frequency of stress testing to document ischemia prior to elective percutaneous coronary intervention. JAMA. 2008;300:1765-1773.

5. Melikian N, De Bondt P, Tonino P, et al. Comparison between visua assessment and quantitative angiography versus fractional flow reserve for native coronary narrowing of moderate severity. Am J Cardiol. 2002;90:210-215

6. Christou MA, Siontis GC, Katristris DG, Ioannidis JP. Meta-analysis of fractional flow reserve versus quantitative coronary angiography and noninvasive imaging for evaluation of myocardial ischemia. Am J Cardiol. 2007;99:450-456.

7. Tonino PA, de Bruyne $\mathrm{B}$, Pijls $\mathrm{NH}$, et al. Fractional flow reserve versus angiography for guiding percutaneous coronary intervention. $N$ Eng/ J Med. 2009;360:213-224.

8. Bourdarias JP. Coronary reserve: concept and physiological variations. Eur Heart J. 1995;16(suppll):2-6

9. Jeremias A, Whitbourn RJ, Filardo SD, et al. Adequacy of intracoronary versus intravenous adenosine-induced maximal coronary hyperemia for fractional flow reserve measurements. Am Heart J. 2000;140:651-657.

10. Pijls $\mathrm{NH}$,Tonino PA, Fearon WF. Fractional flow reserve for guiding $\mathrm{PCl} . \mathrm{N}$ Engl J Med. 2009;360:2026-2027.

11. Vranckx P, Cutlip DE, Mc Fadden EP, Kern MJ, Mahran R, Muller O Coronary pressure derived fractional flow reserve measurements: recommendations for standardization, recording and reporting as a core laboratory technique. Proposal for integration in clinical trials. Circ Cardiovasc Interv. 2012;5:312-317.

12. Tonino PA, Fearon WF, De Bruyne B, et al. Angiographic versus functional severity of coronary artery stenosis in the FAME study fractional flow reserve versus angiography in multivessel evaluation. J Am Coll Cardiol. 2010;55:2816-2821.

13. Lilly LS, Harvard Medical School. Pathophysiology of heart disease: a collaborative project of medical students and faculty. $5^{\text {th }}$ ed. Baltimore/ MD: Wolters Kluwer Lippincott Williams \& Wilkins; 2011

14. Leonardi RA, Townsend JC, Patel CA, et al. Left ventricular end-diastolic pressure affects measurement of fractional flow reserve. Cardiovasc Revasc Med. 2013;14:218-222.

15. De Bruyne B, Piljs NH, Kalesan B, et al. Fractional flow reserve - guided $\mathrm{PCl}$ versus medical therapy in stable coronary disease. $N$ Engl J Med. 2009;360:213-224.

16. De Bruyne B, Piljs NH, Barbato $\mathrm{E}$, et al. Intracoronary and intravenous adenosine 5-triphosphate, adenosine, papaverine and contrast medium to assess fractional flow reserve in humans. Circulation. 2003;107:18771883.

17. Yong AS, Daniels D, De Bruyne B, et al. Fractional flow reserve assessment of left main stenosis in the presence of downstream coronary stenoses. Circ Cardiovasc Interv. 2013;6:161-165.

18. Bech GJ, de Bruyne $\mathrm{B}$, Piljs $\mathrm{NH}$, et al. Fractional flow reserve to determine the appropiatenes of angioplasty in moderate coronary stenosis; a randomized trial. Circulation. 2001;103:2928-2934.

19. De Bruyne B, Piljs NH, Bartunek J, et al. Fraxctional flow reserve in patients with prior myocardial infarction. Circulation. 2001;104:157-162.

20. Pijls $\mathrm{NH}$. Fractional flow reserve to guide coronary revascularization. Circ J. 2013;77:561-569

21. Van Belle E, Rioufol G, Pouillot C, et al. Outcome impact of coronary revascularization strategy reclassification with fractional flow reserve at time of diagnostic angiography: insights from a large French multicenter fractional flow reserve registry. Circulation. 2014;129:173-185
22. Melikian N, Cuisset T, Hamilos M, De Bruyne B. Fractional flow reserve the influence of the collateral circulation. Int J Cardiol. 2009;132:e109-e110.

23. Wijns W, Kohl P, Danchin N, et al. Guidelines on myocardial revascularization: The Task Force on Myocardial Revascularization of the European Society of Cardiology (ESC) and the European Association for Cardio-thoracic Surgery (EACTS). Eur Heart J. 2010;31:2501-2555.

24. Koo BK, Park KW, Kang HJ, et al. Physiological evaluation of the provisional side branch intervention strategy for bifurcation lesion using fractional flow reserve. Eur Heart J. 2008;29:726-732.

25. Koo BK. Physiologic evaluation of bifurcation lesions using fractional flow reserve. J Interv Cardiol. 2009;22:110-113.

26. Fearon WF, Shilane D, Piljs NH, et al. Cost-effectiveness of percutaneous coronary intervention in patients with stable coronary artery disease and abnormal fractional flow reserve. Circulation. 2013:128:1335-1340.

27. Ntalianis A, Sles JW, Davidavicius G, et al. Fractional flow reserve for the assessment of nonculprit coronary artery stenoses in patients with acute myocardial infarction. JACC Cardiovasc Interv. 2010;3:1274-1281.

28. Levine GN, Bates ER, Blankenship, et al. 2011 ACCF/AHA/SCAl Guideline for Percutaneous Coronary Intervention: a report of the American College of Cardiology Foundation/American Heart Association Task Force on Practice Guidelines ant the Society for Cardiovascular Angiography and Interventions. Circulation. 2011;124:e574-651.

29. Fearon WF. Percutaneous Coronary Intervention Should Be Guided by Fractional Flow reserve Measurement. Circulation. 2014;129:1860-1870.

30. Burzotta F, Leone AM, De Maria GL, et al. Fractional flow reserve or optical coherence tomography guidance to revascularize intermediate coronary stenosis using angioplasty (FORZA) trial: study protocol for a randomized controlled trial. Trials. 2014;15:140.

31. Meuwissen M, Chamuleau SA, Siebes M, et al. Role of variability in microvascular resistence on fractional flow reserve and coronary blood velocity reserve in intermediate coronary lesions. Circulation. 2001;103:184-187.

32. Sen S, Escaned J, Malik IS, et al. Development and validation of a new adenosine-independent index of stenosis severity from coronary waveintensity analysis: results of the ADVISE (ADenosine Vasodilator Independent Stenosis Evaluation) study. J Am Coll Cardiol. 2012:59:1392-1402.

33. Berry C, van t Veer M, Witt N, et al. VERIFY (VERification of Instantaneous Wave-Free Ratio and Fractional Flow Reserve for the Assessment of Coronary Artery Stenosis Severity in EverydaY Practice): a multicenter study in consecutive patients. J Am Coll Cardiol. 2013:61:1421-1427.

34. Escaned J, Echavarria-Pinto M, Garcia-Garcia HM, et al. Prospective Assessment of the Diagnostic Accuracy of Instantaneous Wave-Free Ratio to Assess Coronary Stenosis Relevance: Results of ADIVSE II International, Multicenter Study (ADenosine Vasodilator Independent Stenosis Evaluation II). JACC Cardiovasc Interv. 2015;8:824-833.

35. Nijjer SS, Sen S, Petraco R, et al. Pre-angioplasty instantaneous wave-free ratio pullback provides virtual intervention and predicts hemodynamic outcome for serial lesions and diffuse coronary artery disease. JACC Cardiovasc Interv. 2014:7:1386-1396.

36. Van Belle E. DEFINE REAL: a prospective, observational, non-randomised, European, multicentre registry, collecting real-life information for the utilisation of iFR in assessing coronary stenosis relevance in the multivessel disease patient population. Presented at: EuroPCR 2016.

37. Stone GW, Maehara A, Lansky AJ, et al. A prospective natural-history study of coronary atherosclerosis. N Engl J Med. 2011;364:226-235.

38. Johnson NP, Gould KL, Di Carli MF, Taqueti VR. Invasive FFR and Noninvasive CFR in the Evaluation of Ischemia: What Is the Future? J Am Coll Cardiol. 2016:67:2772-2788

39. Nijjer SS, Sen S, Petraco R, et al. Improvement in coronary haemodynamics after percutaneous coronary intervention: assessment using instantaneous wave-free ratio. Heart. 2013;99:1740-1748

40. Fearon WF, Aarnoudse W, Pijls NH, et al. Microvascular resistance is not influenced by epicardial coronary artery stenosis severity: experimenta validation. Circulation. 2004;109:2269-2272.

41. De Bruyne B, Barbato E. Quantitative assessment of the coronary microvasculature: new tools for the black box. Circulation. 2013;127:23782379.

42. Fearon WF, Low AF, Yong AS, et al. Prognostic value of the Index of Microcirculatory Resistance measured after primary percutaneous coronary intervention. Circulation. 2013;127:2436-2441. 